\title{
Exploiting Stochastic Petri Net formalism to capture the Relapsing Remitting Multiple Sclerosis variability under Daclizumab administration.
}

\author{
Simone Pernice \\ Department of Computer Science, \\ University of Torino, \\ Torino, Italy \\ pernice@di.unito.it
}

\author{
Greta Romano \\ Department of Computer Science, \\ University of Torino, \\ Torino, Italy \\ grromano@unito.it
}

\author{
Marzio Pennisi \\ Department of Mathematics and Computer Science, \\ University of Catania, \\ Catania, Italy \\ mpennisi@dmi.unict.it
}

\author{
Giulia Russo \\ Department of Drug Sciences, \\ University of Catania, \\ Catania, Italy \\ giulia.russo@unict.it
}

\author{
Francesco Pappalardo \\ Department of Drug Sciences, \\ University of Catania, \\ Catania, Italy \\ francesco.pappalardo@unict.it
}

\begin{abstract}
It is well known that the response of individuals to disease varies, either because of unpredictable exogenous events, such as possibly unknown environmental effects, or just because of endogenous factors, i.e. different genetic background. In particular, when a treatment effectiveness has to be validated, the individual variability should be taken into account by exploiting stochastic models. Relapsing Remitting Multiple Sclerosis (RRMS) is an unpredictable and complex disease, whose random behaviour perfectly fits the study with stochastic models. RRMS is the most common form of Multiple Sclerosis (MS), an immunemediated inflammatory disease of the central nervous system, characterized by alternate episodes of symptom exacerbation (relapses) with periods of disease stability (remission). Several treatments were proposed to contrast the disease progression. Among these, Daclizumab initially exhibited promising results. However, due to the risk of serious side effects the treatment has been retired. We propose a stochastic and an hybrid extension, based on a generalization of the high level Petri Net formalism, of an existing model of Daclizumab effects on RRMS. The model is developed to investigate the complex mechanisms and unpredictable behaviour characterizing the RRMS disease and its relapsing, especially under the Daclizumab administration.

Index Terms-Multiple Sclerosis, Computational modeling, Stochastic modeling, Petri Net.
\end{abstract}

\section{INTRODUCTION}

When a new treatment is designed, to be allowed for sale from the respective regulatory agency, two aspects have to be demonstrated: 1) its safety: it will not worsen the disease course or give rise to undesirable side effects; and 2) its effectiveness: it will improve the recipient health. Indeed, the verification of such features can be achieved through specific experiments executed under controlled conditions, with the involvement of several patients. These experiments,

978-1-7281-1867-3/19/\$31.00 @2019 IEEE usually referred to as clinical trials, are very expensive but fundamental to assess the usefulness of a treatment at the population level. In other words, it must be assured that the treatment is both safe and effective independently from the biological, immunological, epigenetic and environmental differences characterizing the individuals involved into the trial.

More recently, many regulatory agencies are opening to the possibility of using computational methods to verify the treatments safety and effectiveness, in particular through the use of large scale simulations on virtual patients. These experiments, referred to as "in silico" trials, will open to new possibilities in the design and experimentation of novel treatments, with a reduction of time and costs. Besides their capability of reproducing the disease dynamics and the effects of a given treatment, such "in silico" models must also demonstrate to be able to capture individual diversity. In many instances people diversity can be described and introduced inside the models through a vector of biological features, but in other cases, due to the lack of information about the disease, or simply because of unpredictable environmental events, some of the individual variability can be captured only by exploiting stochastic approaches. An example of such diseases, with an only partially understood etiology, is Multiple Sclerosis (MS). MS is a chronic and potentially highly disabling disease with considerable social impacts and economic consequences. In Europe it is the leading cause of non-traumatic disabilities in young adults, since more than 700,000 EU people suffer from MS [1].

The scientific community commonly agrees that MS involves a process mediated by immune system in which an abnormal response of the bodys immune defense is directed against the central nervous system (CNS), made up of the brain, spinal 
cord and optic nerves. Within the CNS, the immune system causes an inflammation process that damages the myelin (i.e. the fatty substance that surrounds and insulates the nerve fibers), the nerve fibers themselves and the specialized cells that make myelin (i.e. Oligodentrocytes, ODC). The direct consequence of damaging in MS patients is the alteration or completely interruption of nervous messages within the $\mathrm{CNS}$, producing a variety of neurological symptoms that will vary among people with MS in type and severity. As already stated, the causes of MS are still not very clear, but physicians retain that they include abnormalities in the immune system and environmental factors (e.g. Epstein-Barr Virus) that combine to trigger the disease [2]. People with MS typically experience one of four disease patterns (types of MS) with a predominance of the Relapsing-Remitting MS (RRMS) course observed in approximately $85 \%$ of patients at diagnosis [2]. RRMS patients alternate episodes of symptom exacerbation (relapses) with periods of disease stability with complete or partial recovery (remission) [1]. Until now, dozen treatments have been proposed to stop, slow and contrast the RRMS progression. Such disease modifying therapies include oral agents and monoclonal antibodies, which have been designed for a selectivity of the drug action. Daclizumab (DAC) is one of the therapeutic monoclonal antibody directed with high-affinity against interleukin-2 receptor (IL2R). IL2R is the receptor-structure able to bind a key component of the immune system, Interleukin 2 (IL2). Daclizumab introduces a new mechanism of action preventing the binding of IL2 with its receptor with a consequent blocking of immune regulation and its effect on a population of cells with regulatory ability, such as the Natural Killer (NK) cells [2]. DAC efficacy was demonstrated in reducing the clinical relapse rate of RRMS, the disability progression and in improving health-related quality of life in patients with relapsing MS [2]. Daclizumab appears to be generally well tolerated by the patients but with some adverse events as infections, encephalitis, and liver damages. For these reasons Daclizumab has been withdrawn from the market worldwide.

The study and the simulation of MS involves a series of hypotheses about the disease mechanisms that cannot be always described as deterministic parameters to be included into an "in silico" model. Environmental events, the complex balance mechanism between the Treg and Teff cells, or the random spreads of self-reactive $\mathrm{T}$ cells cannot be well predicted, and fall within that part of uncertainty that cannot be quantified and that can potentially distinguish the disease course of different individuals, even with the same genetic background. In this perspective, some stochastic models of RRMS have been presented. Vélez de Mendizábal et al. [3] presented a Stochastic Differential Equations (SDE) model to reproduce the typical oscillating behavior of RRMS. In [4] the authors present a (stochastic) agent based model with a more complete description of the entities involved into the disease, including some thoughts about the role of vitamin D [5], and the Blood Brain Barrier [6].

Differently, in [7]-[9] a computational methodology is proposed to analyse the RRMS behaviour exploiting the deterministic approximation of the model based on a system of ordinary differential equations. Indeed, the deterministic process is not capable to reproduce relevant random features like variance, bimodality, and tail behaviour. For these reasons, with the purpose to improve our understanding of this complex disease, we will extend the RRMS models presented in these works for the stochastic analysis.

Hence, we firstly describe how Extended Stochastic Symmetric Net (ESSN) [9], [10] formalism can be efficiently used to derive a graphical and parametric description of the system under study. Then, we show how the Master equations, that can be automatically derived from an ESSN model, well describe the stochastic behaviour of this disease. In particular, when complex models are studied, the computational cost of the stochastic simulation could become significantly high. To cope with this the so called Hybrid models [11], [12], where parts of model are considered deterministic and others stochastic, can be exploited to obtain a good approximation of the system behaviour reducing computational cost.

Then, to show the relevance to consider the stochasticity and variability into a biological system, we applied such methodology to the RRMS model introduced in [8], [9].

In conclusion, in this work we firstly describe how the ESSN formalism can be efficiently used to derive a graphical and parametric description of the system under study, and secondly how to obtain automatically the Master equations, which reproduce the stochastic disease dynamics. Finally we test the proposed methodology constructing two different models, the former completely stochastic and the latter hybrid, which allow us to analyse the variability characterizing the Daclizumab administration considering a diseased RRMS patient.

\section{BACKGROUND}

In this section we introduce the high-level Petri Net extension called Extended Stochastic Symmetric Net (ESSN) [9], which is used to model and analyze our case study. Then, we report how the stochastic behaviour of a system modeled through such a formalism can be efficiently computed by exploiting the Gillespie algorithm, and by a hybrid approach. Finally, we briefly recall the biological system characterizing the Multiple Sclerosis and its respective ESSN model, rigorously defined in [8], [9].

\section{A. Extended Stochastic Symmetric Net}

Petri Nets (PNs) [13] and their extensions are widely recognized to be a powerful tool for modeling and studying biological systems thanks to their ability of representing systems in a graphical manner and of allowing the computation of qualitative and quantitative information about the behavior of these systems. Among the PN generalisations proposed in literature, Extended Stochastic Symmetric Nets (ESSNs) [9], [10] extend the PN formalism providing a more compact, parametric, and readable representation of the system.

In details, ESSNs are bipartite directed graphs with two types of nodes, the former are called places and are graphically 
represented as circles, the latter are called transitions and are graphically represented as boxes. Usually, places correspond to the state variables of the system, and the transitions to the events that might occur. Two different nodes are connected by arcs, which express the relation between states and event occurrences. To each arc is associated a specific cardinality (multiplicity), describing the number of tokens, objects contained in each place and drawn as black dots, whose can be removed from (or added to) the corresponding place. In ESSN, it is possible to associate specific information (i.e. colors) with each token, making the model more compact and parametric. Then, the number of colored tokens in each place defines the state of an ESSN, called marking.

We define a transition enabled, if and only if each input place contains a number of tokens greater or equal than a given threshold defined by the cardinality of the corresponding input arcs. Thus, the firing of an enabled transition removes a fixed number of tokens from its input places and adds a fixed number of tokens into its output places, according to the cardinality of its input/output arcs. Hence, to allow the definition of complex rate functions, the set of transitions $T$ is split in two sub-sets $T_{m a}$ and $T_{g}$, distinguishing the standard transitions, which fire with a rate following a Mass Action (MA) law, and the general transitions, whose random firing delay have rates that are defined as general real functions that might depend only from the time and the corresponding input places. The standard transitions are graphically represented by white bars, while the general ones by black bars.

An example of a simple ESSN is given in Fig. 1, where it is represented the activation, and so the entrance to system, of the Effector T cells (Teff) due to the Epstain Bar Virus (EBV) occurrence. The Teff cells start to attack both the virus cells and the myelin sheaths, expressed by the Oligodentrocytes (ODC), given the structure similarity of the viral protein with myelin proteins. This is a sub net of the more complex ESSN represented in Fig. 2, for this reason further details will be given in Sec.II-C. This sub net is characterized by three places, $T e f f, E B V$ and $O D C$, representing the Effector T cells, Epstain Bar Virus cells, and the Oligodentrocytes cells, respectively. This model is defined by one standard transition representing the Teff cells death, namely TeffDeath, and by four general transitions modeling: the virus occurrence, i.e. the activation of the Teff cells, the control mechanism given by the Teffs over the EBV cells, and finally the damage of the mylen sheets, respectively called $E B V$ injection, TeffActivation, TeffKillsEBV, and TeffKillsODC.

To associate colors with the tokens, we have to define a color domain to each place $p$, denoted $c d(p)$. Color domains are defined by the Cartesian product of elementary types called color classes, $\mathcal{C}=\left\{C_{1}, \ldots, C_{n}\right\}$, which are finite and disjoint sets, and might be further partitioned into (static) subclasses. Similarly, a color domain is associated with transitions and is defined as a set of typed variables, where the variables are those appearing in the functions labeling the transition arcs and their types are the color classes. Then, we can define an instance, denoted as $\langle t, c\rangle$, of a given transition $t$ as an assignment (or binding) $c$ of the transition variables to a specific color of a proper type. Moreover, we define guard a logical expression defined on the color domain of the transition, which can be used to define restrictions on the allowed instances of a transition. For instance, in the example in the Fig. 1 the $O D C$ color domain is defined by one color class, the myelination levels of ODC cells, named Mye. This is divided into 5 static subclasses (i.e. $L_{\min }, L 1, L 2, L 3$ and $L_{\max }$ ) so that myelination level ranges from an irreversible damage ( $L_{\min }$, no myelination) to no damages $\left(L_{\max }\right.$, full myelination). So, the color domain of TeffKillsODC transition, representing the damage of an ODC cell, is Mye and the variable characterizing its input arc is $l \in M y e$.

Each ESSN arc is labeled with an expression defined by the function $I[p, t]$, if the arc connects a place $p$ to a transition $t$, or $O[p, t]$ for the opposite direction. The evaluation of $I[p, t]$ (resp. $O[p, t]$ ), given a legal binding of $t$, provides the multiset of colored tokens that will be withdrawn from (input arc) or will be added to (output arc) the place connected to that arc by the firing of such transition instance. Moreover, we denote with ${ }^{\bullet} \mathbf{t}$ and $\mathbf{t}^{\bullet}$ the set of input and output places, respectively, of the transition $t$. We use the notation $E(t, m)$ to denote the set of all instances of $t$ enabled in marking $m$. Where, in the case of ESSN formalism, a transition instance $\langle t, c\rangle$ is enabled and can fire in an marking $m$, if: (1) its guard evaluated on $c$ is true; (2) for each place $p$ we have that $I[p, t](c) \leq m(p)$, where $\leq$ is the comparison operator among multisets. The firing of the enabled transition instance $\langle t, c\rangle$ in $m$ produces a new marking $m^{\prime}$ such that, for each place $p$, we have $m^{\prime}(p)=$ $m(p)+O[p, t](c)-I[p, t](c)$.

In ESSNs each transition is associated with a specific rate, representing the parameter of the exponential distribution that simulates its firing time. So, let define $\hat{m}(\nu)=\left.m(\nu)\right|^{\bullet} \mathbf{t}$ as the subset of the marking $m(\nu)$ concerning only the input places to the transition $t$. Then, the parameter associated with an enabled transition instance $\langle t, c\rangle$ is given by the function

$$
F(\hat{m}(\nu), t, c, \nu):=\left\{\begin{aligned}
\varphi(\hat{m}(\nu), t, c), & t \in T_{m a}, \\
f_{\langle t, c\rangle}(\hat{m}(\nu), \nu), & t \in T_{g}, \\
f_{\langle t, c\rangle} & \in \Omega(t, c)
\end{aligned}\right.
$$

where $\Omega=\left\{f_{\langle t, c\rangle}\right\}_{t \in T \wedge c \in c d(t)}$ is set grouping all the real functions characterizing the transition speeds $\forall t \in T$, with $f_{\langle t, c\rangle}=\varphi(\cdot, t, c)$ when $t \in T_{m a}$. Where $\varphi(m(\nu), t, c)$ is the MA law, i.e.

$$
\varphi(m(\nu), t, c)=\omega(t, c) \prod_{\left\langle p_{j}, c^{\prime}\right\rangle \mid} m_{p \in \bullet \mathbf{t} \wedge c^{\prime} \in c d\left(p_{j}\right)}
$$

with $\omega(t, c)$ the rate of the enabled transition instance $\langle t, c\rangle$. Observe that $\varphi(\hat{m}(\nu), t, c)$ and $f_{\langle t, c\rangle}(\hat{m}(\nu), \nu)$ can depend only on the time $\nu$ and the marking of the input places of transition $t$ at time $\nu$. Stochastic firing delays, sampled from a negative exponential distribution, allow one to automatically derive the underlying Continuous Time Markov Chain 
(CTMC) that can be studied to quantitatively evaluate the system behaviour [13]. In details, the CTMC state space, $\mathbb{S}$, corresponds to the reachability set of the corresponding ESSN, i.e. all possible markings that can be reached from the initial marking. Thus, the Master equations for the CTMC are defined as follows:

$$
\frac{d \pi\left(m_{i}, \nu\right)}{d \nu}=\sum_{m_{k}} \pi\left(m_{k}, \nu\right) q_{m_{k}, m_{i}} \quad m_{i}, m_{k} \in \mathbb{S}
$$

where $\pi\left(m_{i}, \nu\right)$ represents the probability to be in marking $m_{i}$ at time $\nu$, and $q_{m_{k}, m_{i}}$ the velocity to reach the marking $m_{i}$ from $m_{k}$, defined as

$$
q_{m_{k}, m_{i}}=\sum_{\substack{\mathbf{t} \in T \wedge \\ T\left\langle\mathbf{t}, \mathbf{c}^{\prime}\right\rangle \in E\left(\mathbf{t}, m_{k}\right)_{\mid m_{i}}}} F\left(m_{k}, \mathbf{t}, \mathbf{c}^{\prime}, \nu\right)\left(L[p, \mathbf{t}]\left(\mathbf{c}^{\prime}\right)[c]\right) .
$$

Where $E\left(\mathbf{t}, m_{k}\right)_{\mid m_{i}}$ is the set of all instances of $\mathbf{t}$ enabled in marking $m_{k}$ whose firing bring to the marking $m_{k}$, and $L[p, \mathbf{t}]\left(\mathbf{c}^{\prime}\right)[c]=O[p, \mathbf{t}]\left(\mathbf{c}^{\prime}\right)[c]-I[p, \mathbf{t}]\left(\mathbf{c}^{\prime}\right)[c]$.

In complex systems these equations 2 are often compuatationally intractable, then several techniques, such as the Monte Carlo simulation, can be exploited to study the system taking into account stochasticity.

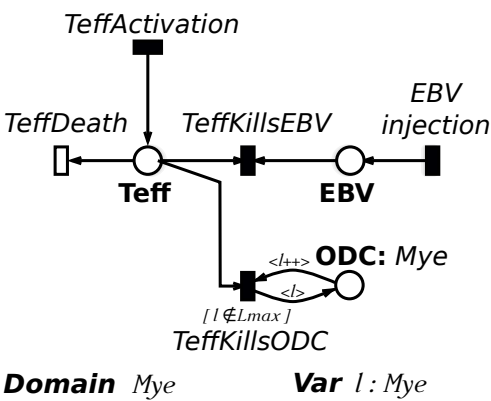

Class Mye $=$ circular $\{l l\}$ is $L \min +\{12\}$ is $L 2+\{13\}$ is $L 3+\{14\}$ is $L 4+\{15\}$ is $L$ max

Fig. 1: Sub net of ESSN Fig. 2, representing the appearance of the EBV in the system, the consequent activation of Teff cells and the damage the ODCs.

\section{B. Stochastic Simulation}

Deterministic approximation, in which the system behaviour is approximated by a deterministic model described through an Ordinary Differential Equations (ODEs) system, is one of the most used approach for studying a dynamical system. However, in those systems in which randomness plays an important role this approach is able to provide a good approximation of the real system behaviour. To deal with these systems stochastic models can be instead exploited.

In 1976 Daniel Gillespie proposed an innovative algorithm, called Stochastic Simulation Algorithm (SSA) [14], to simulate chemical or biochemical systems of reactions. The SSA is an exact stochastic method to simulate chemical systems, indeed it is possible to obtain sample trajectories distributed according to the solution of the Master equations, eq.s 2. Since this method explicitly simulates all the events that might occur in the system, it becomes often slow when the number of system molecules increased. For this reason several algorithms [15]-[17] were proposed for obtaining similar approximations as the SSA ones, but with significantly lower computational costs. One of the most common of these approximate simulation algorithms is the $\tau$-leaping algorithm [16]. Indeed by using a Poisson approximation the $\tau$-leaping algorithm can leap over many fast reactions and approximate the stochastic behavior of the system very well. Then it provides a natural connection between the SSA in the discrete stochastic regime and the explicit Euler method applied in the continuous deterministic approximation.

Differently, hybrid simulation is based on the combination of discrete and continuous variables into the same model, by defining which transitions have to be considered stochastic or deterministic. Then, the hybrid simulation co-simulates the discrete (stochastic) part of the model and the continuous (deterministic) ones. In particular, the discrete components of the model are treated according to the mechanisms of the original CTMC, eq. 2, and are simulated by exploiting the SSA algorithm components. While the continuous variables are approximated by solving an ODEs system. How to obtain the ODEs system underlying an ESSN model is rigorously explained in [9]. Thus, the resulting process is a hybrid process where the discrete state changes can be seen as switches that take the ODE simulation from one condition to another.

Indeed, this approach is an approximation of the stochastic process, and by choosing the right events (transitions) that have to be considered as continuous, a significant reduction of the computational time and a better approximation on the system behavior with respect to the deterministic simulation can be obtained. However, in this approach the choice of which transitions must be dealt as continuous ones is critical. For instance, the modeling of fast transitions [12] (i.e. transitions firing several times in one simulation step) such as discrete ones might return a hybrid approximation worthless compared to the stochastic simulation, since the resolution time might considerably increase due the multiples switches from one process to the other. Therefore, a pre-analysis on the net to identify the fast transitions, and so to set them as continuous variables, i.e. deterministic, could be crucial.

\section{A model of Relapsing-Remitting Multiple Sclerosis}

RRMS is an autoimmune disease in which the immune system of the patient react against itself removing the myelin sheath (i.e. Oligodentrocyte (ODC)) from the nerve fibers of the CNS preventing the efficient transmission of the nervous signals. Although the precise cause of RRMS has not yet been established, the disease is understood to result from an abnormal immune-mediated response triggered by environmental factors in genetically susceptible individuals. In particular, viruses may play a role in MS pathogenesis acting as such environmental triggers [18]. An hypothesis is that the Epstein Barr Virus (EBV) first infection as well as the reactivation of the latent infection can cause the activation of auto-reactive $\mathrm{T}$ effector (Teff) lymphocytes against the CNS 
resulting in damage to myelin and nerve fibers, and triggering an inflammatory cascade. Teff lymphocytes produce IL2, an immunomodulating molecule, released for a self-stimulation of Teff to duplicate and propagate their actions, via the binding of IL2 to the receptor IL2R, located on the surface of the cells [18]. In this context, another type of lymphocytes, $\mathrm{T}$ regulatory (Treg) and Natural Killer cells (NK) give also a contribute regulating the auto-reactive Teff cells activity and acting as first-line defense against the virus, respectively. However, despite the continuous attacking action of Teff to myelin results in a reduction of the same, it is worth to note that in some cases the ODC are able to partially recover the lost myelin if the damage is not excessive.

All these cellular interactions characterizing the RRMS are described by the model showed in Fig. 2. The model consists of 10 places and 22 transitions, four are standard and 18 general. The corresponding general functions are rigorously reported in [9]. We can distinguish seven modules corresponding to the biological entities which play a key role in the development of the disease, as illustrated in Fig. 2.

Firstly, the EBV module, characterized by the place $E B V$, simulates the virus injections in the system through the EBVinj transition. Then the Treg module, constituted by the Resting_Treg and Treg places, encodes the arrival of new resting Treg cells from thymus, the control mechanism of the Treg over the Teff, and their activation, proliferation and death, respectively by the following transitions: FromTimoREG, TregKillsTeff, TregActivation, TregDup and TregDeath. Similarly to this module is the third module regarding the Teff cells, with the addition of the EffectorMemory place. In particular, the annihilation of the virus by the Teff action is encoded by the TeffKillsEBV, while the duplication transition is split into two different transitions, namely TeffDup_Sym and TeffDup_Asym, simulating the Teff cell proliferation which might take place in two different manners called Symmetrical and Asymmetrical processes. The fourth module is defined by one place corresponding to the NK cells. Through the transition NKentry is simulated the new NK cells arrival, and by the NKKillsTeff and NKKillsTreg transitions the killing of self-reactive Teff and Treg cells, respectively. Finally, the death and proliferation of the NK cells are modeled by transition NKDeath and NKdup. The IL2 module focuses on the role of this protein, and it is characterized by IL 2 place. The module encodes the IL 2 consumption during the Treg, Teff and $N K$ proliferation, and its production from the Teff activation. The ODC module describes instead the ODC behaviour, characterized particularly by the damage caused by Teff cells on ODC cells, represented by the TeffKills $O D C$ transition. To simulate this damage, we associate with the place $O D C$ the color class Mye encoding the myelination levels of ODC. Mye is divided into five static subclasses ranging from L $\min$ (no myelination) to L $\max$ (full myelination). Indeed, when the myelin level reaches the lowest value, an irreversible damage occurs and a remyelinization, given by the Remyelinization transitions, of the neurons is no more possible. Finally, the DAC module encodes the drug administration, modeled through the place $D A C$, and its pharmacokinetics inhibition of the expansion of Treg and Teff, decreasing the velocity of transitions TregDup,TeffDup_Sym, and TeffDup_Asym. The drug administration is modeled by transition DACinj, and its degradation by DACDegradation.

\section{RESULTS}

In this section we firstly present the prototype computational framework that we developed to study the RRMS. Then, we discuss the results obtained from the stochastic and hybrid model, in which we investigated the effect of the Daclizumab administration in MS patient. In details, all the constants and parameters used were obtained from [9].

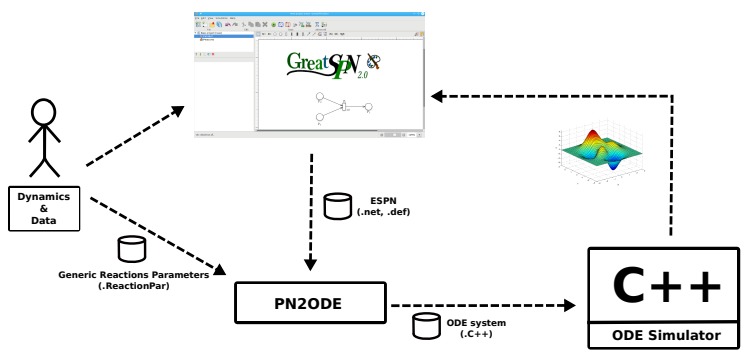

Fig. 3: Architecture of the prototype framework combining GreatSPN suite with $\mathrm{C}++$.

\section{A. Framework Architecture}

The framework that we developed and proposed for studying the RRMS disease is integrated in GreatSPN [19], a wellknown software suite for modeling and analyzing complex systems through the PN formalism and its generalizations. The architecture of this framework is depicted in Fig. 3. In details, by exploiting GreatSPN, a Java Graphic User Interface (GUI), it is possible to draw and build the ESSN model, then calling PN2ODE, a C/C++ program, the underlying stochastic and deterministic processes are generated. Then, the processes underlying the model are automatically generated and saved into a binary file with .solver extension, from which deterministic, hybrid, or stochastic simulation algorithm can be settled. Let us note that the type of transitions, such as general and stochastic, can be defined dynamically adding a specific label to the transition through the GreatSPN GUI. Observe that file storing the functions corresponding to the general transitions is optional and it is needed only when a rate function different from the one specified by Mass Action law is used. Finally, let us underline that the GreatSPN GUI can be downloaded at https://github.com/greatspn; instead, all the $\mathrm{C} / \mathrm{C}++$ files and the GreatSPN files of the net are freely available at https://github. com/qBioTurin/ESSNandRRMS/tree/master/StochasticModel.

\section{B. Stochastic Model}

In this work we report only the trajectories related to the $O D C$ and $N K$ places, since they are the most important variables for identifying the spreading of the disease and the therapy effectiveness. In this experiment for the missing input parameters we used the value estimated in [9], so that RRMS patient is characterized by an irreversible damage in 


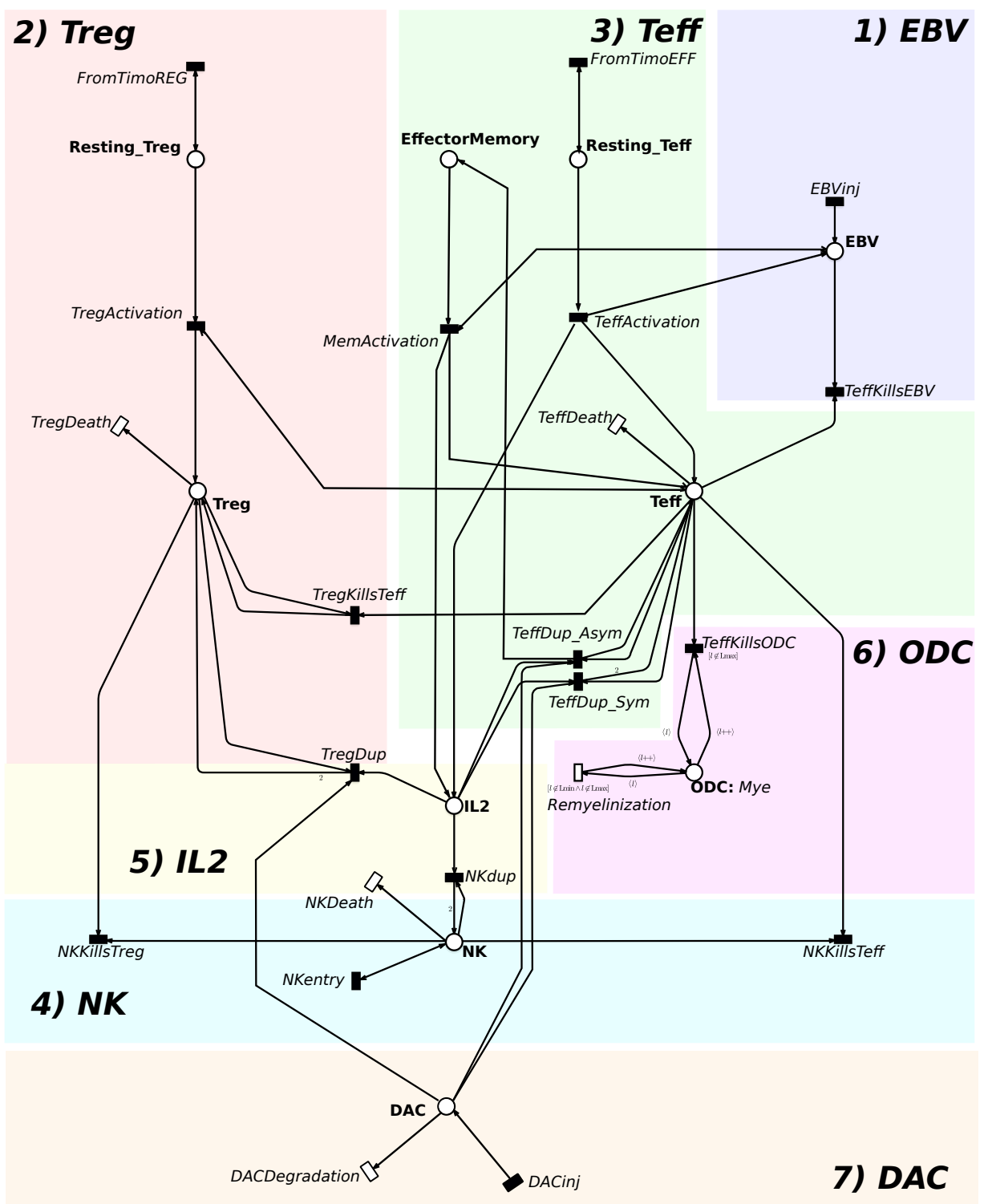

Fig. 2: RRMS Model represented by exploiting the ESSN graphical formalism.

about $80 \%$ of ODC cells. Therefore, starting with a value of $500 O D C$ cells with the highest level of neuronal myelinization, we define the disease occurrence when the lowest level of neuronal myelinization is reached for each ODC cell. This event represents an irreversible damage. For all the simulations, we assumed as initial marking the following parameters consistent with a space of $1 \mathrm{~mm}^{3}$ of blood and $4 \mathrm{~mm}^{3}$ of neural tissue: 500 ODC with level $L_{\max }$ of neuronal myelinization, 1687 resting Teff cells, 63 resting Treg cells, 375 NK cells and 1000 IL2 molecules, and zero cells in the other places (see [9]). In particular, we modeled firstly the developing of the disease (the Fig. 4's left column, namely "Without therapy"), and successively the Daclizumab therapy administrated by using the same initial conditions (the Fig. 4's right column, namely "With therapy"). In details, both the scenarios are characterized by five virus injections at irregular times, introducing into the system 1000 EBV copies per injection. Then, only for the second scenario we simulated the DAC administration, one each two months, at the 53rd day after the first EBV injection, introducing in the system 30 DAC molecules (it has been shown in [9] that the amount of DAC administered does not worthily impact the result). In these experiments, showed in Fig. 4, 500 trajectories (in grey) were simulated by exploiting the SSA algorithm over one year time interval $[0,364]$. Thus, it is possible to see that in first scenario, both the mean trends (blue lines) of the irreversibly ODC cells damaged and NK cells, calculated from the 500 simulations, are very similar to the deterministic 
solution (red line) obtained from [9]. This clearly highlights that the deterministic process is a good approximation, and the randomness of the system can be disregarded in this phase. Considering the Daclizumab therapy administration on the same model, it is straightforward to see the disease relapse with a decreasing to $40 \%$ of ODCs irreversibly damaged, Fig. 4c), but with an increasing mean trend of the NK cells with respect to deterministic one, Fig. 4d). In particular, comparing Fig. 4b) and Fig. 4d) is possible to see that with the therapy we have in both the cases (deterministic and stochastic) an increasing of the NK cells, as expected from literature [2]. Since the stochastic trajectories in Fig. 4d) are characterized by a most increasing trend, with a mean value quite different from the ODEs solution, we can conclude that the stochastic model is probably more suitable to capture the side effects characterizing the Daclizumab treatment.

\section{Hybrid Model}

Similarly to the experiments showed above, here we show the simulations obtained considering an hybrid model instead of a completely stochastic one. Since we are interested to analyze the variability on the therapy outputs, we decide to fix as stochastic transitions: 1) the ones related to the DAC therapy, i.e. TregDup, TeffDup_Asym, TeffDup_Sym and DACDegradation transitions, and 2) the the remyelinization of the ODC cells, i.e. Remyelinization transition. It is straightforward to observe similar results for the ODC place Fig. 5ac), but instead a complete loss of the stochasticity for the NK place is present, as shown in Fig. 5b-d). Although we estimated a decrease of the computational time, especially considering the scenario with the Daclizumab administration, from an average of $67.02 \mathrm{~s}$. for the stochastic model to $23.23 \mathrm{~s}$. for the hybrid model, a loss of stochastic information regarding the NK cells variability is visible, even if we can still observe from Fig. 5d) an increasing of the NK cells with respect to Fig. 5b), and so the different mean trend could mislead the choice of the model to exploit.

\section{CONClusion ANd FUture PERspective}

In this paper we proposed two modeling approaches for the analysis of the RRMS variability with the Daclizumab administration. In particular, the descriptive power of Extended Stochastic Symmetric Nets is exploited to provide a graphical, parametric and compact representation of this disease. Then we showed that the model is able to reproduce some of the variability that usually characterizes this disease and its Daclizumab treatment, showing in particular that not always a deterministic model, or an hybrid model, is able to effectively capture all the effects entitled with the use of a given therapy.

We remind here that, from an "in silico" trial perspective, it mandatory to consider the potential variability that is involved with different individuals, and not only the mean behavior entitled with the use of a given compound. A treatment must be effective for the majority of, if not all, the patients involved, and thus the capability of reproducing such variability is fundamental to test and optimize through the use of computational models both dosage and timing.

As future works we will implement the $\tau$-leaping algorithm able to include the general functions characterizing the ESSN model. Currently the Gillespie algorithm and hybrid simulation are the only Stochastic solvers available to simulate an ESSN model, due to the assumptions on which the $\tau$ leaping approach is based: (i) the calculation of the partial derivatives of the rate functions or (ii) the hypothesis that all the reactions have to follow the Mass Action law. Such constraints could be obviously not respected by the ESSN model, given the presence of general functions, and so the resulting difficulties for the partial derivatives calculation. Therefore, with an improving of the time simulation, which does not affect the the goodness of the results, we plan to encode in the stochastic model the spatial coordinates of all entities in a cubic tissue portion.

\section{REFERENCES}

[1] Dutta, R., Trapp, B.D.: Mechanisms of Neuronal Dysfunction and Degeneration in Multiple Sclerosis. Prog. Neurobiol. 93(1), 1-12 (2011)

[2] Shirley, M.: Daclizumab: a review in relapsing multiple sclerosis. Drugs 77(4), 447-458 (2017)

[3] Vélez de Mendizábal, N., Carneiro, J., Solé, R.V., Goñi, J., Bragard, J., Martinez-Forero, I., Martinez-Pasamar, S., Sepulcre, J., Torrealdea, J., Bagnato, F., Garcia-Ojalvo, J., Villoslada, P.: Modeling the effector - Regulatory $\mathrm{T}$ cell cross-regulation reveals the intrinsic character of relapses in Multiple Sclerosis. BMC Systems Biology 5 (2011)

[4] Pennisi, M., Rajput, A.M., Toldo, L., Pappalardo, F.: Agent based modeling of treg-teff cross regulation in relapsing-remitting multiple sclerosis. In: BMC Bioinformatics, vol. 14(Suppl 16):S9 (2013)

[5] Pappalardo, F., Pennisi, M., Rajput, A.-M., Chiacchio, F., Motta, S.: Relapsing-Remitting Multiple Scleroris and the role of Vitamin D : an agent based model. In: ACM-BCB, pp. 744-748 (2014)

[6] Pennisi, M., Russo, G., Motta, S., Pappalardo, F.: Agent based modeling of the effects of potential treatments over the bloodbrain barrier in multiple sclerosis. Journal of Immunological Methods (2015)

[7] Beccuti, M., Cazzaniga, P., Pennisi, M., Besozzi, D., Nobile, M.S S. Pernice, Russo, G., Tangherloni, A., F., P.: Gpu accelerated analysis of treg-teff cross regulation in relapsing-remitting multiple sclerosis. In: 4th International European Conference on Parallel and Distributed Computing (Euro-Par 2018). LNCS. Springer, ??? (2018)

[8] Pernice, S., Beccuti, M., Do', P., Pennisi, M., Pappalardo, F.: Estimating daclizumab effects in multiple sclerosis using stochastic symmetric nets. In: IEEE International Conference on Bioinformatics and Biomedicine, BIBM 2018, Madrid, Spain, December 3-6, 2018, pp. 1393-1400 (2018)

[9] Pernice, S., Pennisi, M., Romano, G., Maglione, A., Cutrupi, S., Pappalardo, F., Balbo, G., Beccuti, M., Cordero, F., Calogero, R.A.: A computational approach based on the colored petri net formalism for studying multiple sclerosis. BMC bioinformatics, To be published (2019)

[10] Pernice, S., Follia, L., Balbo, G., Sartini, G., Totis, N., Lió, P., Merelli, I., Cordero, F., Beccuti, M.: Integrating petri nets and flux balance methods in computational biology models: a methodological and computational practice. Fundamenta Informaticae, To be published (2019)

[11] Pola, G., Bujorianu, M.L., Lygeros, J., Di Benedetto, M.D.: Stochastic hybrid models: An overview. IFAC Proceedings Volumes 36(6), 45-50 (2003)

[12] Haseltine, E.L., Rawlings, J.B.: Approximate simulation of coupled fast and slow reactions for stochastic chemical kinetics. The Journal of chemical physics 117(15), 6959-6969 (2002)

[13] Marsan, M.A., Balbo, G., Conte, G., Donatelli, S., Franceschinis, G.: Modelling with Generalized Stochastic Petri Nets. J. Wiley, New York, NY, USA (1995)

[14] Gillespie, D.T.: Exact stochastic simulation of coupled chemical reactions. The journal of physical chemistry 81(25), 2340-2361 (1977)

[15] Gibson, M.A., Bruck, J.: Efficient exact stochastic simulation of chemical systems with many species and many channels. The journal of physical chemistry A 104(9), 1876-1889 (2000) 

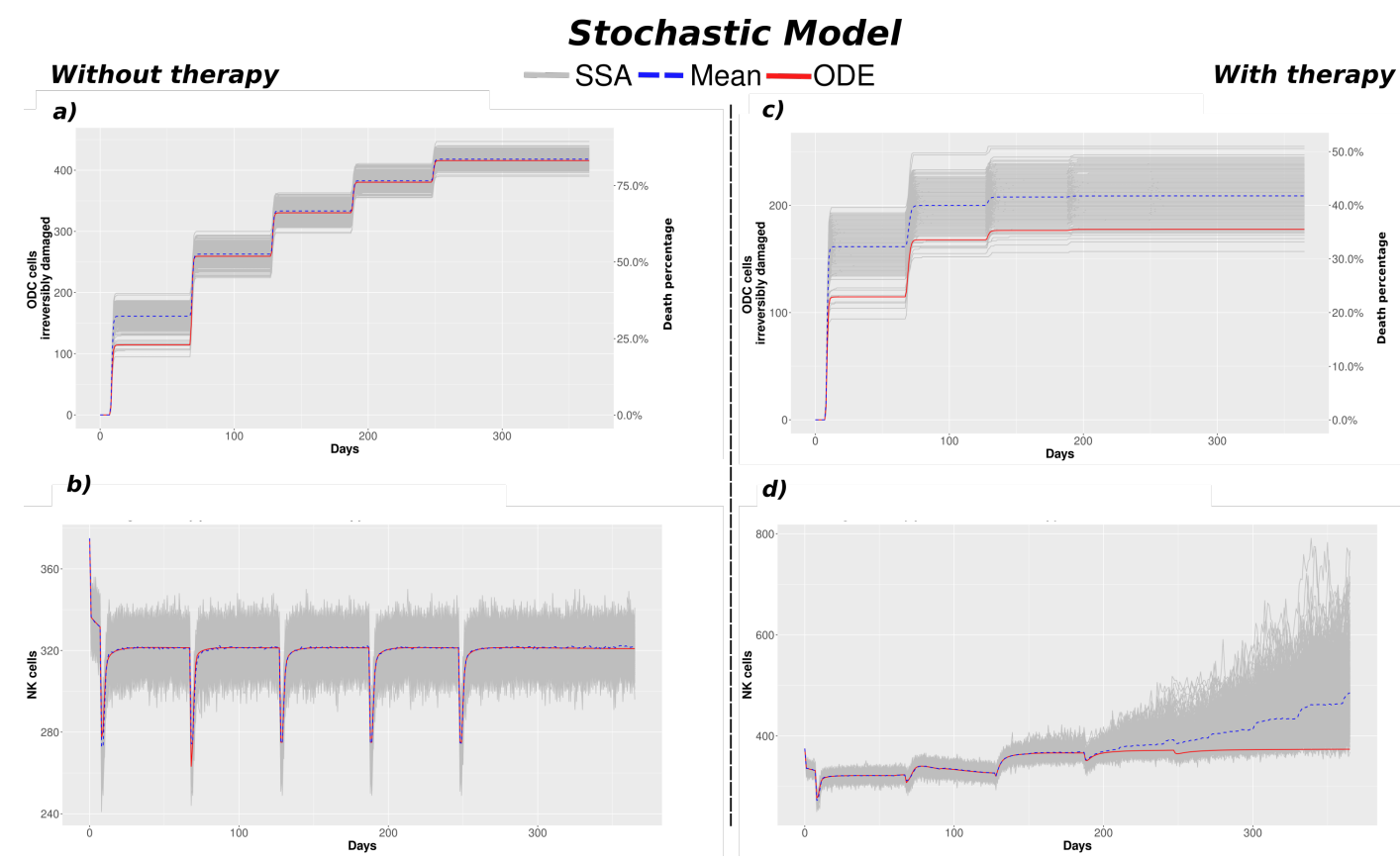

Fig. 4: 500 trajectories (grey) considering the stochastic model of the ODC cells with an irreversible damage (a-c), and the NK cells (b-d) over the whole time interval for both with and without Daclizumab administration. The blue dashed line represents the mean trend, and the red line the deterministic solution of the ODEs system.
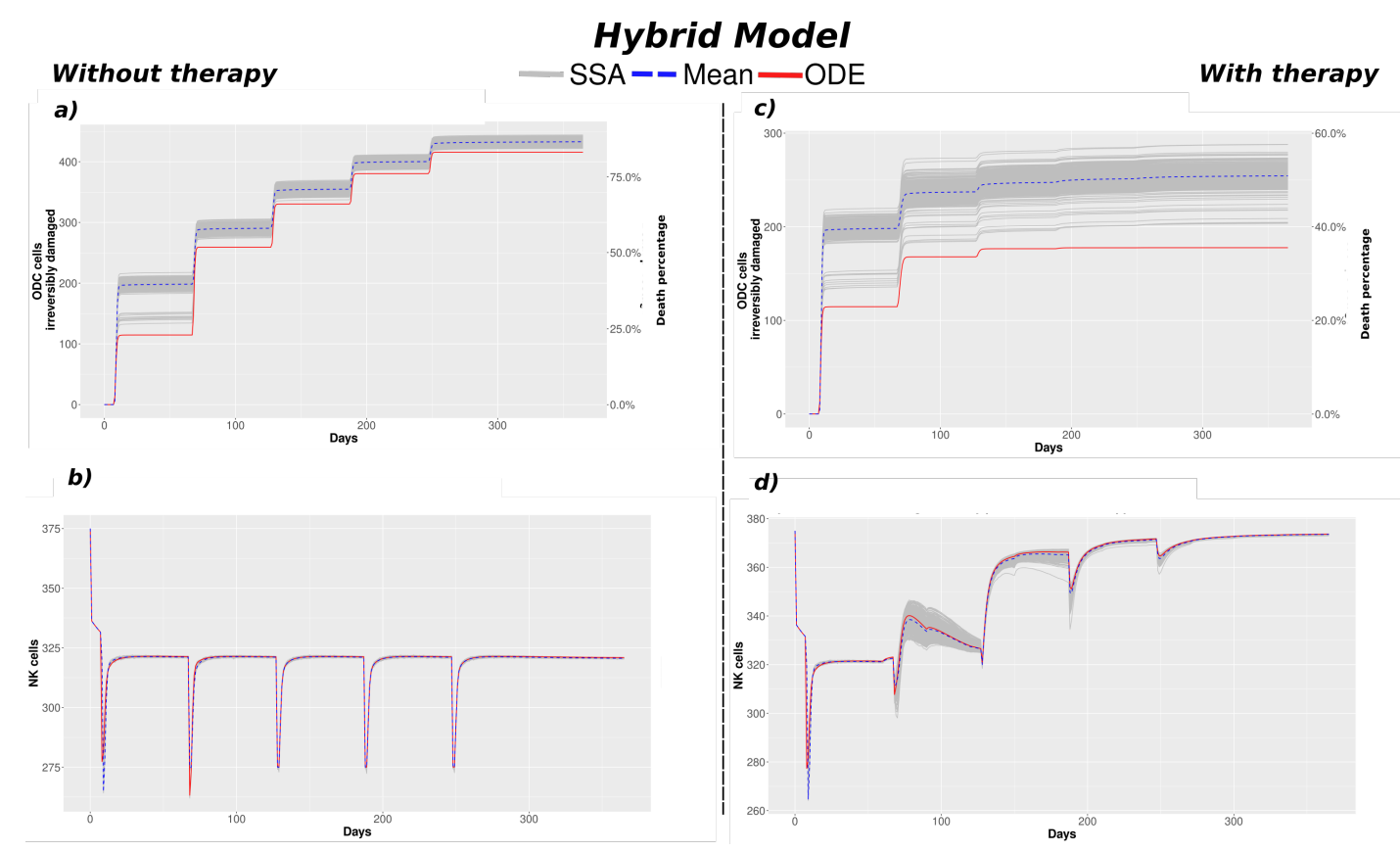

Fig. 5: 500 trajectories (grey) considering the hybrid model of the ODC cells with an irreversible damage (a-c), and the NK cells (b-d) over the whole time interval, with and without Daclizumab administration. The blue dashed line represents the mean trend, and the red line the deterministic solution of the ODEs system.

[16] Gillespie, D.T.: Approximate accelerated stochastic simulation of chemically reacting systems. The Journal of Chemical Physics 115(4), 1716$1733(2001)$

[17] Cao, Y., Li, H., Petzold, L.: Efficient formulation of the stochastic simulation algorithm for chemically reacting systems. The journal of chemical physics 121(9), 4059-4067 (2004)
[18] Virtanen, J.: Viruses and Multiple Sclerosis. CNS Neurol Disord Drug Targets (5), 528-544 (2012)

[19] Babar, J., Beccuti, M., Donatelli, S., Miner, A.S.: GreatSPN enhanced with decision diagram data structures. In: Lilius, J., Penczek, W. (eds.) Application and Theory of Petri Nets. PETRI NETS 2010. LNCS, vol. 6128, pp. 308-317 (2010) 